UCOFIS $1 / 02$

June 2002

\title{
A simple model for nuclear structure functions at small $x$ in the dipole picture
}

\author{
N. Armesto \\ Departamento de Física, Módulo C2, Planta baja, Campus de Rabanales, \\ Universidad de Córdoba, E-14071 Córdoba, Spain
}

\begin{abstract}
A simple model for nuclear structure functions in the region of small $x$ and small and moderate $Q^{2}$, is presented. It is a parameter-free extension, in the Glauber-Gribov approach to nuclear collisions, of a saturation model for the nucleon. A reasonable agreement with experimental data on ratios of nuclear structure functions is obtained. Nuclear effects in the longitudinal-to-transverse cross section ratios are found to be small. Predictions of the model for values of $x$ smaller than those available to present experiments are given. The unintegrated gluon distribution and the behaviour of the saturation scale which result from this model are shown and discussed.
\end{abstract}




\section{Introduction}

The differences between the structure functions measured in nucleons and nuclei [1], the so-called EMC effect, are a very important feature for the study of nuclear structure and nuclear collisions. At small values of the Bjorken variable $x(\lesssim 0.01$, shadowing region), the structure function $F_{2}$ per nucleon turns out to be smaller in nuclei than in a free nucleon. The nature of this shadowing is well understood qualitatively: In the rest frame of the nucleus, the incoming photon splits, at high enough $Q^{2}$, into a $q \bar{q}$ pair long before reaching the nucleus, and this $q \bar{q}$ pair interacts with it with typical

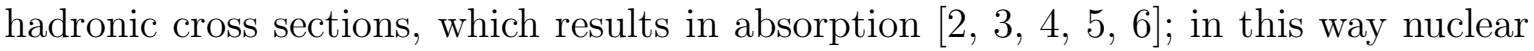
shadowing is a consequence of multiple scattering and is thus related with diffraction [7]. An equivalent explanation in the frame in which the nucleus is moving fast, is that gluon recombination due to the overlap of the gluon clouds from different nucleons, makes gluon density in nucleus with mass number $A$ smaller than $A$ times that in a free nucleon [8, 9]. These studies have received great theoretical impulse with the development of semiclassical ideas in QCD and the appearance of non-linear equations for evolution in $x$ in this framework [10, 11, 12, 13, 14, 15, 16], although saturation appears to be different from shadowing [17] (see [18] for a simple geometrical approach in this framework).

On the other hand, a different approach is taken in [19]: parton densities inside the nucleus are parametrized at some scale $Q_{0}^{2} \sim 2 \mathrm{GeV}^{2}$ and then evolved using the DGLAP [20] evolution equations. In this way, the origin of the differences of partons densities in nucleons with respect to nuclei is not addressed, but contained in the parametrization at $Q_{0}^{2}$ which is obtained from a fit to experimental data.

The results from different models usually depend on additional semiphenomenological assumptions and often contradict each other. For example, concerning the $Q^{2}$-dependence of the effect, in [3, 因, 汭 it is argued that $q \bar{q}$ configurations of a large dimension give the dominant contribution to the absorption, which results essentially independent of $Q^{2}$ (this is the case in [6] until extremely small $x$, where a dependence $\propto \ln Q / Q$ appear related with the use of BFKL evolution [21]). On the other hand, in the gluon recombination approach of [9] the absorption is obtained as a clear highertwist effect dying out at large $Q^{2}$. Finally, in the models [19] which use DGLAP, all $Q^{2}$-dependence comes from QCD evolution and is thus of a logarithmic, leading-twist nature, see [22] for a comparison between multiple scattering and DGLAP approaches. 
Predictions (particularly for the gluon density) on the $x$-evolution towards small $x$ turn out to be very different 23, 24].

In practice these studies are of uttermost importance to compute particle production in collisions involving nuclei. For example, in the framework of collinear factorization 25] parton densities in the nucleus following the spirit of [19 are needed, see e.g. 26] for recent applications to inclusive particle production in heavy ion collisions. While

this scheme is suitable to compute particle production at scales $\Lambda_{Q C D}^{2} \ll Q^{2} \lesssim s$ and thus in the hard region, for semihard (minijet) production, $\Lambda_{Q C D}^{2} \ll Q^{2} \ll s$, the $k_{T^{-}}$ factorization scheme [27] should become the suitable one (in 28, 29, 30 applications to heavy ion collisions can be found). Here the tool that is needed is the so-called unintegrated gluon distribution (see a precise definition below and 31 for a review of its situation for nucleons). Let us stress that different approaches give very different predictions for multiplicities at RHIC and LHC [32].

In this paper we present a simple model for nuclear structure functions in the region of small $x(\lesssim 0.01)$ and of small and moderate $Q^{2}\left(\lesssim 20 \mathrm{GeV}^{2}\right)$ in the dipole picture [3, 33. It consists on an extension to nuclei, using the Glauber-Gribov picture [34, of the saturation model for the proton in [35] without any new parameter (in [30] a similar strategy is used but with a simplified dipole-nucleon cross section and no comparison with experimental data on nuclear structure functions is performed; also in [16 this ansatz is used but just as an initial condition for a non-linear evolution equation). The plan of the paper is as follows: In the next Section the model will be described. In Section 3 a comparison of the results of the model with available data on $F_{2 A}$ will be shown. In Section 1 the unintegrated gluon density obtained in this model, together with the saturation scale it implies, will be discussed. Finally, in the last Section conclusions and possible applications of the model will be outlined.

\section{Description of the model}

The nuclear structure function $F_{2}$ can be standardly defined via the cross sections $\sigma_{T, L}$ for the collision of the transversal $(T)$ or longitudinal $(L)$ virtual photon of momentum $q, q^{2}=-Q^{2}$, on the nucleus $A$ of momentum $A p$ :

$$
F_{2 A}\left(x, Q^{2}\right)=\frac{Q^{2}}{\pi e^{2}}\left(\sigma_{T}^{A}+\sigma_{L}^{A}\right) ;
$$


this expression holds for the small $x$ region where we are going to work. Both cross sections can be conveniently presented in the dipole model [3, 33] via the cross section $\sigma_{d A}(x, r)$, for the scattering of a colour dipole of transverse size $r$ on the nucleus:

$$
\sigma_{T, L}^{A}(x)=\int d^{2} r \rho_{T, L}(r) \sigma_{d A}(x, r)
$$

where $\rho_{T, L}$ are the distributions of colour dipoles created by splitting of the incident photon into $q \bar{q}$ pairs $[3]$ :

$$
\rho_{T}(r)=\frac{e^{2} N_{c}}{8 \pi^{3}} \sum_{f} z_{f}^{2} \int_{0}^{1} d \alpha\left\{\left[\alpha^{2}+(1-\alpha)^{2}\right] \epsilon^{2} \mathrm{~K}_{1}^{2}(\epsilon r)+m_{f}^{2} \mathrm{~K}_{0}^{2}(\epsilon r)\right\}
$$

and

$$
\rho_{L}(r)=\frac{e^{2} N_{c}}{2 \pi^{3}} Q^{2} \sum_{f} z_{f}^{2} \int_{0}^{1} d \alpha \alpha^{2}(1-\alpha)^{2} \mathrm{~K}_{0}^{2}(\epsilon r) .
$$

Here summation goes over flavours (which will be limited to 3 or 4 , see the comments below Eq. (6) $), \epsilon^{2}=Q^{2} \alpha(1-\alpha)+m_{f}^{2}$, and $m_{f}$ and $z_{f}$ are respectively the mass and electric charge in units of the proton charge $e$, of the quark of flavour $f$.

For the total cross section of a dipole on a proton we will use the saturation model in [35], which provides a good description of inclusive and diffractive experimental data on $F_{2 p}$ for $x<0.01$ and small and moderate $Q^{2}<20 \mathrm{GeV}^{2}$. Other models which also describe data in this region could be used, as that in 36], see [37 for a comparison between this model and that of [35], which we will employ because of its simplicity. The form of the cross section is

$$
\sigma_{d p}(x, r)=\sigma_{0}\left[1-\exp \left(-\frac{Q_{s}^{2}(x) r^{2}}{4}\right)\right]
$$

with

$$
Q_{s}^{2}(x)=Q_{0}^{2}\left(\frac{x_{0}}{\tilde{x}}\right)^{\lambda}, \quad \tilde{x}=x\left(1+\frac{4 m_{f}^{2}}{Q^{2}}\right) .
$$

In 35 some parameters were kept fixed: $Q_{0}^{2}=1 \mathrm{GeV}^{2}, m_{u}=m_{d}=m_{s}=0.14 \mathrm{GeV}$ and $m_{c}=1.5 \mathrm{GeV}$. Three parameters were determined from a fit to data and have values $\sigma_{0}=23.03(29.12) \mathrm{mb}, \lambda=0.288(0.277)$ and $x_{0}=3.04 \cdot 10^{-4}\left(0.41 \cdot 10^{-4}\right)$ for the 3 -flavour (4-flavour) version of the model. $Q_{s}^{2}$ is called the saturation scale, which we will discuss in Section 4 .

\footnotetext{
${ }^{1}$ In this model no impact parameter of the proton is explicitly given, so it cannot be considered a pure eikonalization of some elementary amplitude 17; on the contrary, in our extension to nuclei, Eqs. (7) and (8) below, the impact parameter of the nucleus is explicitly taken into account.
} 
Let us comment that, neglecting the influence of quark masses in variable $\epsilon$ which has 38 small effect in the case of light quarks, this model implies, in its 3-flavour version, an exact scaling of the cross sections $\sigma_{T, L}^{A}$ with $\tau=Q^{2} / Q_{s}^{2}$. This scaling has been shown [38] to be fulfilled to a good approximation by all DIS data for $x<0.01$ (which go up to $Q^{2} \sim 450 \mathrm{GeV}^{2}$ ) $)^{2}$. So this model, which leads for large $Q^{2}$ to Bjorken scaling, is apparently able to mimic along a wide $Q^{2}$-region the QCD evolution (see [39] for improvements of this model to include DGLAP evolution). This $\tau$-scaling has been argued to hold for such a large $Q^{2}>Q_{s}^{2}\left(\sim 1 \mathrm{GeV}^{2}\right.$ in the region where data are available) in the framework of semiclassical, high-density QCD models [40], and has also been found in numerical solutions of the non-linear evolution equations at small $x$ [13, 15. Let us also point that this model does not correspond to any fixed twist, see 41] for a study of its twist structure.

The extension of this model to the nuclear case can be made in a straightforward manner in the Glauber-Gribov approach [34]: ignoring isospin effects which are negligible at small $x$ where the model will be applied, we will substitute $\sigma_{d p}(x, r)$ by

$$
\sigma_{d A}(x, r)=\int d^{2} b \sigma_{d A}(x, r, b)
$$

with $b$ the impact parameter of the center of the dipole relative to the center of the nucleus and

$$
\sigma_{d A}(x, r, b)=2\left[1-\exp \left(-\frac{1}{2} A T_{A}(b) \sigma_{d p}(x, r)\right)\right]
$$

the total dipole-nucleus cross section for fixed impact parameter, with $\sigma_{d p}(x, r)$ given by Eqs. (5) and (6). $T_{A}(b)$ is the nuclear profile function (longitudinal integral of the nuclear density, $\left.T_{A}(b)=\int_{-\infty}^{\infty} d z \rho_{A}(z, \vec{b})\right)$ normalized to unity, $\int d^{2} b T_{A}(b)=1$; we employ a nuclear density in the form of a 3-parameter Fermi distribution with parameters taken from [42]. With this normalization we recover the dipole-nucleon cross section making a power expansion for small $A T_{A}(b) \sigma_{0}$, keeping the first term and putting $A=1$. Also the centrality (impact parameter) dependence of the structure functions can be computed by direct substitution of $\sigma_{d A}(x, r, b)$, Eq. (8), into Eq. (2). This model implies a new scaling for nucleus of the type of the $\tau$-scaling for proton and a new saturation scale, which we will discuss in Section 4. These two Eqs. (7) and (8) constitute the central point in our extension of the model of [35] to the nuclear case.

\footnotetext{
${ }^{2}$ In the 4-flavour version, a flavour dependence is introduced in $Q_{s}^{2}$ through variable $\tilde{x}$ and some deviation of the scaling may also appear through variable $\epsilon$ in the photon wave function due to the larger charm mass.
} 
Using Eqs. (11)-(8) we can compute the nucleon and nuclear structure functions and the corresponding ratios, which we will compare with experimental data in the next Section. But first let us discuss the region of applicability of the model: This should be that of small $x$ (due to the use of the model of [35] for the nucleon, to the neglection of isospin effects and to the use of (8) which requires a large coherence length, achieved at small $x$, of the photon fluctuation), and of small and moderate $Q^{2}<20 \mathrm{GeV}^{2}$. Although the $\tau$-scaling may suggest that the region of applicability in $Q^{2}$ could be wider, we think that a safe extrapolation to higher $Q^{2}$ would require to implement DGLAP evolution [39]. Also the extrapolation to very small $x$ could imply effects of gluon or pomeron fusion like those included in the non-linear evolution equations mentioned previously, so it should be taken with care. Nevertheless, numerical studies [15] show that the onset of the non-linear effects is quite smooth, becoming large for extremely small $x$. So we consider this model as a reasonable approximation for values of $x \gtrsim 10^{-5} \div 10^{-6}$, which are those relevant for RHIC and LHC. Due to the fact that for nuclear structure functions the amount of experimental data is much more limited than that for nucleons, we will perform the comparison with experimental data for $x<0.02$.

\section{Comparison with experimental data}

Here we show the results of the model together with available experimental data. In Figs. 1-5, the results of the model in the 3-flavour (solid lines) and 4-flavour (dashed lines) versions (see the explanations below Eq. (6)) are compared with experimental data from 443, 44, 45, 46]. In these Figs., $R(A / B)=\left[B F_{2 A}\left(x, Q^{2}\right)\right] /\left[A F_{2 B}\left(x, Q^{2}\right)\right]$. Even when joined with a line, the results of the model have been computed at the same $\left(\langle x\rangle,\left\langle Q^{2}\right\rangle\right)$ as the experimental data; in the latter, the inner error bars are the statistical errors, while the outer error bars show statistical and systematic errors added in quadrature (with overall normalization uncertainties ignored). Considering both the absence of any free parameter to go from the nucleon to the nuclear case and the simplicity of the model, we find the agreement quite reasonable. Concretely, from Figs. 3 and 4 it can be concluded that the $A$ - and $Q^{2}$-dependences of the data at

\footnotetext{
${ }^{3}$ In 15$]$ it is seen that the scaling induced by the non-linear evolution is fulfilled for values of $y=\left[\pi /\left(N_{c} \alpha_{s}\right)\right] \ln \left(x_{0} / x\right)$ greater than 2.2 , which for $\alpha_{s}=0.2$ and $x_{0}=0.01$ means $x$ smaller than $10^{-7}$.
} 
fixed $x$ are well reproduced, while in Figs. 1, 2 and 5 the $x$-dependence is reasonably reproduced, taking into account that these latter Figures contain data with $Q^{2}$ going from $\sim 0.15 \mathrm{GeV}^{2}$ for the smallest $x$ values to $\sim 4.5 \mathrm{GeV}^{2}$ for the highest $x$.

As the version with 3 flavours is simpler, gives an equally reasonable agreement with nuclear data than the 4-flavour version, and produces a better description of the nucleon data [35], from now on we will restrict our computations to the 3-flavour version.

Let us turn now to the behaviour of the ratio of longitudinal to transverse cross sections. Experimentally [47] large nuclear effects have been observed: the ratio $\sigma_{L} / \sigma_{T}$ in $\mathrm{N}\left({ }^{3} \mathrm{He}\right)$ over $\sigma_{L} / \sigma_{T}$ in $\mathrm{D}$ has been found to reach values as high as $\sim 5(\sim 2)$ for $0.01<x<0.03$ and $Q^{2}<1 \mathrm{GeV}^{2}$. Some explanations 48 point to nuclear enhanced power corrections, but the experimental data are under reanalysis [49] and the evidence of such strong nuclear effects is now dubious. While this important point has to be settled, it is clear than in our model such strong effects are not present, as the nuclear effects are contained in the Glauber-Gribov cross section in Eqs. (7) and (8) which is common to both longitudinal and transverse cross sections, see Eq. (2). In Fig. 6 we show the results in our model for the ratio $\sigma_{L} / \sigma_{T}$ in nucleus over $\sigma_{L} / \sigma_{T}$ in proton, for $\mathrm{C}$ and $\mathrm{Pb}$. It can be seen that the nuclear effects never go beyond $\pm 12 \%$, which is a clear prediction of our model.

In Fig. 7 we present predictions of the model for the ratio $F_{2 A} /\left(A F_{2 p}\right)$ for $\mathrm{C}$ and $\mathrm{Pb}$, together with the $x$-evolution for $\mathrm{Be}, \mathrm{C}, \mathrm{Al}, \mathrm{Ca}, \mathrm{Fe}, \mathrm{Sn}$ and $\mathrm{Pb}$ at fixed $Q^{2}=2.25$ $\mathrm{GeV}^{2}$. A clear evolution with $Q^{2}$ can be seen, which in this model is due to the interplay between the (transversal and longitudinal) probabilities to get a dipole of size $r$, Eqs. (3) and (4), and the dipole-target cross section, Eqs. (5), (7) and (8), and cannot be addressed to any concrete twist but to an admixture of all twists, see [41. At large enough $Q^{2}$ this dependence on $Q^{2}$ will eventually disappear, as this model, as stated previously, leads to Bjorken scaling.

As a last point in this Section, let us comment on other possible options to get nuclear structure functions in the framework of the dipole model. A simple form for the dipole-nucleus cross section is suggested by high-density QCD [10, 12, 17]:

$$
\sigma_{d A}(x, r)=\int d^{2} b\left[1-\exp \left(-\frac{Q_{s A}^{2}(b) r^{2}}{4}\right)\right] .
$$

We have tried several relations between $Q_{s A}^{2}$ (the saturation scale in nuclei) and that in proton, $Q_{s}^{2}$. On the one hand, we have used a relation coming from the running of 
the coupling, of the type

$$
Q_{s A}^{2} \ln \left(\frac{Q_{s A}^{2}}{\Lambda_{Q C D}^{2}}\right) \propto\left(\frac{T_{A}(b)}{T_{A}(0)}\right) A^{1 / 3} Q_{s}^{2} \ln \left(\frac{Q_{s}^{2}}{\Lambda_{Q C D}^{2}}\right) .
$$

On the other hand, we have imposed the first scattering approximation (valid for $r \rightarrow 0$ ) in Eq. (8),

$$
Q_{s A}^{2}=\frac{1}{2} A T_{A}(b) \sigma_{0} Q_{s}^{2}
$$

(in this expression the value of the running coupling evaluated at the appropriate scale is hidden in $\sigma_{0}$, see e.g. [17]) 1 . But so far we have not succeeded in getting a satisfactory description of experimental data, not even on a qualitative level: either too strong a shadowing is observed or too fast an evolution in $x$ (and too slow in $Q^{2}$ ) is obtained. Indeed Eqs. (8) and (9) contain different physical assumptions on the nature of the scattering centers: while (8) considers multiple scattering on single nucleons (described by the saturating form (5)), (9) implies scattering on a black area filled with partons coming from many nucleons. Our lack of success in reproducing the experimental data with (9) suggests that higher order rescatterings are actually needed in the exponent of Eq. (5) for the proton, and that the asymptotic region where Eq. (9) should be valid to describe data on $F_{2 A}$ integrated over impact parameter, is not reached yet (i.e. the grey region is still dominating the scattering); a very important test for the form (9) would be its ability to describe experimental data on diffraction (which is indeed fulfilled by (5) 35]). In the next section we will address the behaviour of the saturation scale in our model.

\section{Unintegrated gluon distribution and saturation scale}

As stated in the Introduction, in the $k_{T}$-factorization scheme [27] a key ingredient is the unintegrated gluon distribution of the hadron, $\varphi_{A}(x, k, b)$ (sometimes it appears in

\footnotetext{
${ }^{4}$ The fact that both $Q_{s A}^{2}$ and $Q_{s}^{2}$ may have roughly the same $x$-dependence can be justified by the following qualitative argument: $Q_{s A}^{2}$ is related with $p_{T}$-broadening in nucleus, $Q_{s A}^{2}=n_{A}(b) Q_{s}^{2}$, with $n_{A}(b)$ the number of scatterings at impact parameter $b$. As $n_{A}(b) \propto A T_{A}(b) x G \alpha_{s}\left(Q_{s A}^{2}\right) / Q_{s A}^{2}$ (for a perturbative QCD cross section evaluated at scale $Q_{s A}^{2}$ and $x G$ the gluon distribution in a nucleon) and $Q_{s}^{2} \propto x G$, both $Q_{s A}^{2}$ and $Q_{s}^{2}$ show the same $x$-behaviour (modulo the logarithm coming from the running coupling). Special thanks are given to D. E. Kharzeev for discussions and suggestions on all these points.
} 
the literature as $f=k^{2} \varphi$ [28, 31]), with $k$ the transverse momentum. This $\varphi_{A}(x, k, b)$ at fixed impact parameter $b$ is related, at lowest order in $k_{T}$-factorization [31, to the dipole-nucleus cross section by a Bessel-Fourier transform (see [15, 16]):

$$
\varphi_{A}(x, k, b)=-\frac{N_{c}}{4 \pi^{2} \alpha_{s}} k^{2} \int \frac{d^{2} r}{2 \pi} \exp (i \vec{k} \cdot \vec{r}) \sigma_{d A}(x, r, b),
$$

with $k^{2}=\vec{k} \cdot \vec{k}, r^{2}=\vec{r} \cdot \vec{r}$ and vectors defined in the two-dimensional transverse space. The unintegrated gluon can be related to the 'ordinary' gluon density (that used in collinear factorization [25]) by

$$
x G\left(x, Q^{2}, b\right)=\int^{Q^{2}} d k^{2} \varphi_{A}(x, k, b)
$$

although this expression must be considered with great care, as it is only true for large $Q^{2} \gg Q_{s}^{2}$ (the actual relation is not with the collinear glue but with the gluon distribution in the light-cone wave function of the hadron, see [10, 12, 17]).

For the proton, Eq. (12) leads to the result $\varphi_{p}(x, k) \propto \sigma_{0}\left(k^{2} / Q_{s}^{2}\right) \exp \left(-k^{2} / Q_{s}^{2}\right)$ [35]. For the nucleus, using the technique outlined in the Appendix of [16] (or simply applying $\left[N_{c} /\left(2 \pi^{2} \alpha_{s}\right)\right] k^{2} \nabla_{k}^{2}$ to function $\phi_{0}$ defined in Eqs. (31) and (34) in that Reference), we get

$$
\varphi_{A}(x, k, b)=\frac{N_{c}}{\pi^{2} \alpha_{s}} \frac{k^{2}}{Q_{s}^{2}} \sum_{n=1}^{\infty} \frac{(-B)^{n}}{n !} \sum_{l=0}^{n} C_{n}^{l} \frac{(-1)^{l}}{l} \exp \left(-\frac{k^{2}}{l Q_{s}^{2}}\right),
$$

with

$$
B=\frac{1}{2} A T_{A}(b) \sigma_{0}
$$

As in the case of proton, Eq. (14) shows explicit scaling in $k^{2} / Q_{s}^{2}$; besides, in this Equation (as in (8)) the result for proton is recovered making a power expansion for small $B$, keeping the first term and putting there $A=1$. For realistic values of $B<3$, Eq. (14) turns out to be very suitable for numerical computations, as the convergence of the series in $n$ is very fast and only a few terms are needed to get $\varphi_{A}(x, k, b)$ to the desired accuracy.

In Fig. 8 we show the unintegrated gluon distribution for proton, and for $\mathrm{Pb}$ in three cases: central $(b=0)$, peripheral $(b=7 \mathrm{fm})$, and integrated over $b$, and for two values of $x=10^{-2}$ and $10^{-6}$ (in these computations there is no $Q^{2}$, so the substitution

\footnotetext{
${ }^{5}$ In some proposals [12, 29] it is considered that the unintegrated gluon distribution should tend to a constant as $k \rightarrow 0$. For discussions on the 'correct' definition and behaviour of this quantity, see [15, 30].
} 
in Eq. (6) is meaningless here and we will make no distinction in this Section between $x$ and $\tilde{x}$; this also avoids the complication of flavour dependence in case the 4-flavour version is to be used, although as stated previously we will use the 3-flavour version). A scaling in $k^{2} / Q_{s A}^{2}$ (with $Q_{s A}^{2}$ identified with the position of the maximum, see below) is perfectly visible, as in each case the curves move to the right with decreasing $x$ while keeping their shape and size (this phenomenon has also been found in the framework of the non-linear equations for small $x$ [10, 12, 13, 15] and the solution called a soliton wave [15]). Besides it can be seen that the shape of the curves for different cases is quite close, the only differences being the height, the position of the maximum which at fixed $x$ varies from left to right for proton and $\mathrm{Pb}$ with increasing centrality, and the logarithmic width which slightly increases with increasing centrality (being e.g. at 1/10 the maximum height, 2.20 for proton and 2.40 for central $\mathrm{Pb}$ ).

Keeping in mind the difficulties to identify at small and moderate $Q^{2}$ the integral of the unintegrated gluon distribution with the ordinary gluon density, Eq. (13) (see the comments below it), it is still tempting to use this Equation and compare with other approaches. A comparison at $Q^{2}=5 \mathrm{GeV}^{2}$ of the results of our model with others, for the ratio of gluon densities in $\mathrm{Pb}$ over proton, can be found in [24]. There it can be seen that our results at $x \simeq 10^{-2}$ roughly coincide with those of [19, 50] and are higher than those of [22, 51], while at $x \simeq 10^{-5}$ they become smaller than those of [19, 50], get close to those of [22] and are still larger than those of [51]. Apart from the constraints coming from existing DIS experimental data on nuclei which are very loose for the glue at small $x$, in [19] the saturation of gluon shadowing comes mainly from the initial condition for DGLAP evolution where this saturation has been imposed, while [22, 50] are theoretical models and in [51] the behaviour of the glue has been fixed in order to reproduce charged particle multiplicities in AuAu collisions at RHIC. Additional caution has to be taken to compare our results with those coming from DGLAP analysis [19]: our ratios for the glue at some moderate, fixed $Q^{2}$ and very small $x$ result smaller than the ratios for $F_{2}$ at the same $x, Q^{2}$, which leads to problems with leading-twist DGLAP evolution [23]; as our model mimics the DGLAP increase of $F_{2}$ along a wide $Q^{2}$-region, this is most probably related with the use of Eq. (13) at too small $Q^{2}$. In any case, our model leads to Bjorken scaling at some $Q^{2}$ which increases with decreasing $x$ : the $Q^{2}$-independence of the gluon density is achieved in our model at $Q^{2} \simeq 15 \mathrm{GeV}^{2}$ for $x=10^{-2}$ and at $Q^{2} \simeq 300 \mathrm{GeV}^{2}$ for $x=10^{-6}$. Of course this is due to the exponential decrease of the unintegrated gluon at large $k^{2}$, 
see (14); other proposals (see e.g. [12, 29]) consider a behaviour $\propto 1 / k^{2}$ at large $k^{2}$ which obviously leads to a logarithmic, DGLAP-like increase of the gluon density with increasing $Q^{2}$.

Now we turn to the saturation scale. While for the case of the proton its definition is quite clear in coordinate space, for the nucleus a clean definition is better obtained in momentum space, where it corresponds to the maximum of the unintegrated gluon distribution 112, 13, 15, 52]. The results are shown in Fig. 9 (upper plot) for the same cases as in Fig. 8. Contrary to naive expectations, the saturation scale between proton and central nucleus is not simply proportional to $A^{1 / 3}$ but has a prefactor which makes the result smaller (turning the expected factor 5.9 for $\mathrm{Pb}$ into a factor 2.0). This can be understood using analytical estimates. Taking the exponent in Eq. (8) to be 1/4 (in analogy to the case of the proton), we get

$$
Q_{s A}^{2} \simeq\left[4 \ln \left(\frac{2 A T_{A}(b) \sigma_{0}}{2 A T_{A}(b) \sigma_{0}-1}\right)\right]^{-1} Q_{s}^{2},
$$

which gives a $Q_{s A}^{2} \simeq 2.4 Q_{s}^{2}$ for $\mathrm{Pb}$ at $b=0$. For $A T_{A}(b) \sigma_{0} \gg 1$, we get exactly Eq. (11), recover the expected proportionality $Q_{s A}^{2} \propto A^{1 / 3} Q_{s}^{2}$ and find with this asymptotic form $Q_{s A}^{2} \simeq 2.5 Q_{s}^{2}$. As the form (8) gives a nice agreement with data and (9), (11) do not, we conclude that preasymptotic contributions (i.e. not corresponding to $A T_{A}(b) \sigma_{0} \rightarrow \infty$ ) play a dominant role for the description of present available data.

To perform some comparison with other approaches, let us see the saturation scale coming from the numerical solution of the non-linear evolution equation in [15. There a form (for the evolution obtained starting from a phenomenologically motivated initial distribution) is obtained that can be approximated, for central $(b=0) \mathrm{Pb}$ by the equation

$$
Q_{s A}^{2} \simeq A^{0.37} \exp \left[-6.43+0.78 \ln \left(\frac{x_{0}}{x}\right)\right]
$$

here $x_{0}=0.01$, a value of $\alpha_{s}=0.2$ has been used and the result is in $\mathrm{GeV}^{2}$. The coefficients in this Equation coincide with those obtained in other numerical and analytical studies [12, 13, 40. Its results for $A=1$ and $A=208$ are shown in Fig. 9 (lower plot), but one should keep in mind the way in which the coefficients in Eq. (17) were obtained [15]: They come from a fit to the position of the maximum of the unintegrated gluon distributions for different centralities and values of $x$ but when the scaling induced by the non-linear evolution has already set in. So, from the first footnote it can be concluded that they correspond to $x<10^{-7}$ and thus to a much lower value of $x$ than 
the region where we have actually plotted them in Fig. 9. From the comparison of the two plots in Fig. 9 it is clear that the non-linear evolution produces a much steeper behaviour compared with the model in this paper (i.e. an exponent 0.78 compared to $0.288)$, while the asymptotic $A$-dependence is not really different $(0.37 \simeq 1 / 3)$.

\section{Conclusions}

A simple model for nuclear structure functions in the region of small $x$, and small and moderate $Q^{2}$, has been presented. It is a parameter-free extension of the saturation model for the nucleon of [35] in the Glauber-Gribov approach, although in principle any saturating model which correctly describes nucleon data, e.g. [36, could be used. This simple extension of the model for the nucleus should be valid until non-linear evolution effects [10, 11, 12, 13, 14, 15, 16] become of importance, and for not too high $Q^{2}$ where implementation of DGLAP evolution should be required. Conservative estimates establish the region of validity of the model to be $10^{-5} \div 10^{-6}<x<10^{-2}$ and $Q^{2}<20 \mathrm{GeV}^{2}$.

A reasonable agreement with experimental data is obtained for the $x^{-}, A$ - and $Q^{2}$ dependence. The longitudinal-to-transverse cross section ratios show nuclear effects smaller than $\pm 12 \%$. Predictions of the model for values of $x$ smaller than those available to present experiments have been given.

The unintegrated gluon distribution coming from our dipole-nucleus cross section has been obtained and studied. Besides the behaviour of the saturation scale in this model has been shown and discussed. It turns out to behave $\propto A^{1 / 3}$ for large $A$ as expected, but large prefactors make the resulting scale for nuclei smaller than naive estimates, e.g. for central $\mathrm{Pb} 2$ times bigger, instead of 6 times, than the corresponding scale for proton.

Our model could be used to provide the starting condition for DGLAP evolution, as performed in the approaches of [19], for some initial scale $Q_{0}^{2} \gg \Lambda_{Q C D}^{2}$. The unintegrated gluon distribution could be employed to compute particle production, using the $k_{T}$-factorization scheme [27, 31], in high energy collisions involving nuclei [28, 29, 30]. Work along these directions is in progress.

As a last comment, our study implies the existence of a scaling for nuclei of the same type as that for proton [38], with a non-trivial relation for the $A$-dependence of the saturation scales between both cases, while the $x$-dependence turns out to be 
the same. An experimental, model-independent extraction of the saturation scale in nuclei would be very useful to settle the discussions on the relevance of the semiclassical approach for existing or future experiments, and the region of validity of perturbative QCD and DGLAP evolution. Such an issue would be best explored, and our model tested, in high energy lepton-nucleus colliders 53.

Acknowledgments: The author expresses his gratitude to M. A. Braun, R. Engel, K. Itakura, V. J. Kolhinen, L. McLerran, C. Pajares, P. V. Ruuskanen, R. Venugopalan and U. A. Wiedemann, for useful discussions. Special thanks are given to K. J. Eskola and D. E. Kharzeev for their interest, suggestions and encouragement to write this article, and to A. Capella, E. G. Ferreiro and C. A. Salgado for discussions, suggestions and a critical reading of the manuscript. He also thanks CERN Theory Division, Departamento de Física de Partículas at Universidade de Santiago de Compostela, Department of Physics at University of Jyväskylä, Helsinki Institute of Physics and Physics Department at BNL, for kind hospitality during stays in which parts of this work have been developed. Finally he acknowledges financial support by CICYT of Spain under contract AEN99-0589-C02 and by Universidad de Córdoba.

\section{References}

[1] M. Arneodo, Phys. Rep. 240, 301 (1994); D. F. Geesaman, K. Saito and A. W. Thomas, Ann. Rev. Nucl. Part. Sci. 45, 337 (1995).

[2] S. J. Brodsky, T. E. Close and J. F. Gunion, Phys. Rev. D6, 177 (1972); S. J. Brodsky and H. J. Liu, Phys. Rev. Lett. 64, 1342 (1990).

[3] N. N. Nikolaev and B. G. Zakharov, Z. Phys. C49, 607 (1991).

[4] V. Barone, M. Genovese, N. N. Nikolaev, E. Predazzi and B. G. Zakharov, Z. Phys. C58, 541 (1993).

[5] B. Z. Kopeliovich and B. Povh, Phys. Lett. B367, 329 (1996).

[6] N. Armesto and M. A. Braun, Z. Phys. C76, 81 (1997).

[7] A. Capella, A. B. Kaidalov, C. Merino, D. Pertermann and J. Tran Thanh Van, Eur. Phys. J. C5, 111 (1998). 
[8] L. V. Gribov, E. M. Levin and M. G. Ryskin, Phys. Rept. 100, 1 (1983).

[9] A. H. Mueller and J.-W. Qiu, Nucl. Phys. B268, 427 (1986). J.-W. Qiu, Nucl. Phys. B291, 746 (1987); E. L. Berger and J.-W. Qiu, Phys. Lett. B206, 42 (1988).

[10] L. McLerran and R. Venugopalan, Phys. Rev. D49, 2233 (1994); 3352; D50, 2225 (1994); J. Jalilian-Marian, A. Kovner, L. McLerran and H. Weigert, Phys. Rev. D55, 5414 (1997); E. Iancu, A. Leonidov and L. McLerran, Nucl. Phys. A692, 583 (2001); E. Iancu and L. McLerran, Phys. Lett. B510, 145 (2001); E. G. Ferreiro, E. Iancu, A. Leonidov and L. McLerran, Nucl. Phys. A703, 489 (2002); A. Kovner and U. A. Wiedemann, hep-ph/0112140; preprint CERN-TH-2002-089 (hep-ph/0204277); E. G. Ferreiro, E. Iancu, K. Itakura and L. McLerran, preprint SACLAY-T02/074, NSF-ITP-02-44 (hep-ph/0206241).

[11] I. I. Balitsky, preprint JLAB-THY-97-22 (hep-ph/9706411); Nucl. Phys. B463, 99 (1996).

[12] A. H. Mueller, Nucl. Phys. B335, 115 (1990); B558, 285 (1999); Yu. V. Kovchegov and A. H. Mueller, Nucl. Phys. B529, 451 (1998); Yu. V. Kovchegov, Phys. Rev. D54, 5463 (1996); D55, 5445 (1997).

[13] E. M. Levin and K. Tuchin, Nucl. Phys. B573, 383 (2000); E. M. Levin and M. Lublinsky, Nucl. Phys. A696, 833 (2001); M. Lublinsky, Eur. Phys. J. C21, 513 (2001); K. Golec-Biernat, L. Motyka and A. M. Staśto, Phys. Rev. D65, 074037 (2002).

[14] E. Gotsman, E. M. Levin, U. Maor and E. Naftali, Nucl. Phys. B539, 535 (1999); E. M. Levin and U. Maor, preprint TAUP-2643-2000 (hep-ph/0009217); A. L. Ayala, M. B. Gay Ducati and E. M. Levin, Nucl. Phys. B493, 305 (1997); M. B. Gay Ducati and V. P. Gonçalves, Phys. Lett. B466, 375 (1999).

[15] M. A. Braun, Eur. Phys. J. C16, 337 (2000); hep-ph/0010041; N. Armesto and M. A. Braun, Eur. Phys. J. C20, 517 (2001).

[16] N. Armesto and M. A. Braun, Eur. Phys. J. C22, 351 (2001).

[17] A. H. Mueller, in Proceedings of the XVII Autumn School: QCD: Perturbative or Nonperturbative?, Eds. L. S. Ferreira, P. Nogueira and J. I. Silva-Marcos, World Scientific, Singapore 2001, p. 180. 
[18] N. Armesto and C. A. Salgado, Phys. Lett. B520, 124 (2001).

[19] K. J. Eskola, V. J. Kolhinen and P. V. Ruuskanen, Nucl. Phys. B535, 351 (1998);

K. J. Eskola, V. J. Kolhinen and C. A. Salgado, Eur. Phys. J. C9, 61 (1999); D. Indumathi and W. Zhu, Z. Phys. C74, 119 (1997); M. Hirai, S. Kumano and M. Miyama, Phys. Rev. D64, 034003 (2001).

[20] Yu. L. Dokshitzer, Sov. Phys. JETP 46, 1649 (1977); V. N. Gribov and L. N. Lipatov, Sov. J. Nucl. Phys. 15, 438 (1972); G. Altarelli and G. Parisi, Nucl. Phys. B126, 298 (1977).

[21] V. S. Fadin, E. A. Kuraev and L. N. Lipatov, Phys. Lett. B60 (1975) 50; I. I. Balitsky and L. N. Lipatov, Sov. J. Nucl. Phys. 15 (1978) 438.

[22] L. Frankfurt, V. Guzey, M. McDermott and M. Strikman, JHEP 0202, 027 (2002).

[23] K. J. Eskola, H. Honkanen, V. J. Kolhinen and C. A. Salgado, Phys. Lett. B532, 222 (2002); in Proceedings of the XXXVIIth Rencontres de Moriond: QCD and Hadronic Interactions, Les Arcs, France, March 16th-23rd 2002 (hep-ph/0205231).

[24] C. A. Salgado, talk given at the Second Meeting of the CERN Workshop on Hard Probes in Heavy Ion Collisions at the LHC, Geneve, Switzerland, March 11th-15th 2002 (http://wwwth.cern.ch/lhcworkshop/plenary_mar.html).

[25] J. C. Collins, D. E. Soper and G. Sterman, Nucl. Phys. B261, 104 (1985); B308, 833 (1988); in Perturbative QCD, Ed. A. H. Mueller, World Scientific, Singapore 1989, p. 1.

[26] K. J. Eskola and H. Honkanen, preprint HIP-2002-23/TH (hep-ph/0205048); K. J. Eskola, K. Kajantie, P. V. Ruuskanen and K. Tuominen, Nucl. Phys. B570, 379 (2000); preprint HIP-2002-10-TH, NORDITA-2002-20-HE (hep-ph/0204034).

[27] C. Catani, M. Ciafaloni and F. Hautmann, Nucl. Phys. B366, 135 (1991); J. C. Collins and R. K. Ellis, Nucl. Phys. B360, 3 (1991); E. M. Levin. M. G. Ryskin, Yu. M. Shabelski and A. G. Shuvaev, Sov. J. Nucl. Phys. 53, 657 (1991).

[28] K. J. Eskola, A. V. Leonidov and P. V. Ruuskanen, Nucl. Phys. B481, 704 (1996); M. Gyulassy and L. McLerran, Phys. Rev. C56, 2219 (1997). 
[29] D. E. Kharzeev and M. Nardi, Phys. Lett. B507, 121 (2001); D. E. Kharzeev and E. M. Levin, Phys. Lett. B523, 79 (2001); D. E. Kharzeev, E. M. Levin and M. Nardi, preprint BNL-NT-01/28 (hep-ph/0111315).

[30] H.-J. Pirner and F. Yuan, Phys. Lett. B512, 297 (2001).

[31] B. Andersson et al., preprint DESY 02-041 (hep-ph/0204115).

[32] N. Armesto and C. Pajares, Int. J. Mod. Phys. A15, 2019 (2000).

[33] A. H. Mueller, Nucl. Phys. B415, 373 (1994); A. H. Mueller and B. Patel, Nucl. Phys. B425, 471 (1994).

[34] R. J. Glauber, in Lectures in Theoretical Physics, Vol. 1, Eds. W. E. Brittin and L. G. Duham (Interscience, New York, 1959); V. N. Gribov, Sov. Phys. JETP 29, 483 (1969); 30, 709 (1970).

[35] K. Golec-Biernat and M. Wüsthoff, Phys. Rev. D59, 014017 (1999); D60, 114023 (1999); Eur. Phys. J. C20, 313 (2001).

[36] A. Capella, E. G. Ferreiro, A. B. Kaidalov and C. A. Salgado, Nucl. Phys. B593, 336 (2001); Phys. Rev. D63, 054010 (2001).

[37] M. B. Gay Ducati, E. G. Ferreiro, M. V. T. Machado and C. A. Salgado, Eur. Phys. J. C24, 109 (2002).

[38] A. M. Staśto, K. Golec-Biernat and J. Kwieciński, Phys. Rev. Lett. 86, 596 (2001).

[39] J. Bartels, K. Golec-Biernat and H. Kowalski, preprint DESY-02-037 thepph/0203258).

[40] E. Iancu, K. Itakura and L. McLerran, preprint SACLAY-T02/027 thepph/0203137), to appear in Nucl. Phys. A; S. Munier, hep-ph/0205319.

[41] J. Bartels, K. Golec-Biernat and K. Peters, Eur. Phys. J. C17, 121 (2000).

[42] C. W. De Jager, H. De Vries and C. De Vries, Atom. Data Nucl. Data Tabl. 14, 479 (1974).

[43] E665 Collaboration: M. R. Adams et al., Z. Phys. C67, 403 (1995).

[44] NMC Collaboration: M. Arneodo et al., Nucl. Phys. B481, 3 (1996). 
[45] NMC Collaboration: M. Arneodo et al., Nucl. Phys. B481, 23 (1996).

[46] NMC Collaboration: P. Amaudruz et al., Nucl. Phys. B441, 3 (1995).

[47] HERMES Collaboration: K. Ackerstaff et al., Phys. Lett. B475, 386 (2000).

[48] X.-F. Guo, J.-W. Qiu and W. Zhu, Phys. Lett. B523, 88 (2001).

[49] HERMES Collaboration: A. Bruell et al., in Proceedings of the X International Workshop on Deep Inelastic Scattering (DIS2002), Cracow, Poland, April 30thMay 4th 2002.

[50] Z. Huang, H. J. Lu and I. Sarcevic, Nucl. Phys. A637, 79 (1998).

[51] S.-Y. Li and X.-N. Wang, Phys. Lett. B527, 85 (2002).

[52] A. H. Mueller and D. N. Triantafyllopoulos, preprint CU-TP-1057 hepph/0205167).

[53] M. Arneodo et al., in Proceedings of the Workshop on Future Physics at HERA (Hamburg, Germany, September 1995); H. Abramowicz et al., in TESLA Technical Design Report, Part VI, Chapter 2, Eds. R. Klanner, U. Katz, M. Klein and A. Levy; EIC White Paper, preprint BNL-68933, Eds. A. Deshpande, R. Milner and R. Venugopalan.

\section{Figure captions:}

Fig. 1. Comparison of the results of the model (see text) in the 3-flavour (solid lines) and 4-flavour (dashed line) versions with experimental data at small $x$ for the ratios $\mathrm{C}, \mathrm{Ca}$ and $\mathrm{Pb}$ over $\mathrm{D}$, from 43 .

Fig. 2. Id. to Fig. 1 but with data for $\mathrm{Be}, \mathrm{Al}, \mathrm{Ca}, \mathrm{Fe}, \mathrm{Sn}$ and $\mathrm{Pb}$ over $\mathrm{C}$, from 44.

Fig. 3. Comparison of the $A$-evolution of the results of the model in the 3 -flavour (open circles) and 4-flavour (open triangles) versions with experimental data at small fixed $x$ for $\mathrm{Be}, \mathrm{Al}, \mathrm{Ca}, \mathrm{Fe}, \mathrm{Sn}$ and $\mathrm{Pb}$ over $\mathrm{C}$, from [44].

Fig. 4. Comparison of the $Q^{2}$-evolution of the results of the model in the 3 -flavour (solid lines) and 4-flavour (dashed line) versions with experimental data for Sn over C at small fixed $x$, from [45].

Fig. 5. Id. to Fig. 1 but with data for $\mathrm{C}$ and Ca over D, from 46. 
Fig. 6. Results of the model for the ratio $\sigma_{L} / \sigma_{T}$ in $\mathrm{C}$ and $\mathrm{Pb}$ over $\sigma_{L} / \sigma_{T}$ in proton versus $x$. In the plots, lines going from the bottom to the top correspond to $Q^{2}=0.1$, $0.5,1,2.25,5,10$ and $100 \mathrm{GeV}^{2}$.

Fig. 7. Results of the model for the $x$-dependence of $F_{2 A} /\left(A F_{2 p}\right)$. In the two upper plots, results for $\mathrm{C}$ (upper plot) and $\mathrm{Pb}$ (plot at the middle) versus $x$ are given for $Q^{2}=0.1,0.5,1,2.25,5,10$ and $100 \mathrm{GeV}^{2}$ (lines going from the bottom to the top). In the plot at the bottom, $F_{2 A} /\left(A F_{2 p}\right)$ is drawn versus $x$ at $Q^{2}=2.25 \mathrm{GeV}^{2}$ for $\mathrm{Be}, \mathrm{C}$, $\mathrm{Al}, \mathrm{Ca}, \mathrm{Fe}, \mathrm{Sn}$ and $\mathrm{Pb}$ (lines going from the top to the bottom).

Fig. 8. Upper plot: Results of the model $\left(\right.$ in $\mathrm{GeV}^{-2}$ ) for the unintegrated gluon distribution for proton (solid line) and for $\mathrm{Pb}$ integrated over $b$ (dashed line). Lower plot: Results of the model for the unintegrated gluon distribution for peripheral $(b=7$ fm, solid line) and central ( $b=0$, dashed line) $\mathrm{Pb}$. In each case, two curves are provided for two values of $x=10^{-2}$ (curve to the left) and $10^{-6}$ (curve to the right).

Fig. 9. Upper plot: saturation momentum in the model for proton (solid line), and for $\mathrm{Pb}$ in three cases: central ( $b=0$, dashed-dotted line), peripheral ( $b=7 \mathrm{fm}$, dashed line), and integrated over $b$ (dotted line). Lower plot: saturation momentum in the numerical solution of the non-linear equation in [15], Eq. (17), for $A=1$ (solid line) and $A=208$ (dashed-dotted line). Notice the difference in vertical scales between the plots. 
Figures:
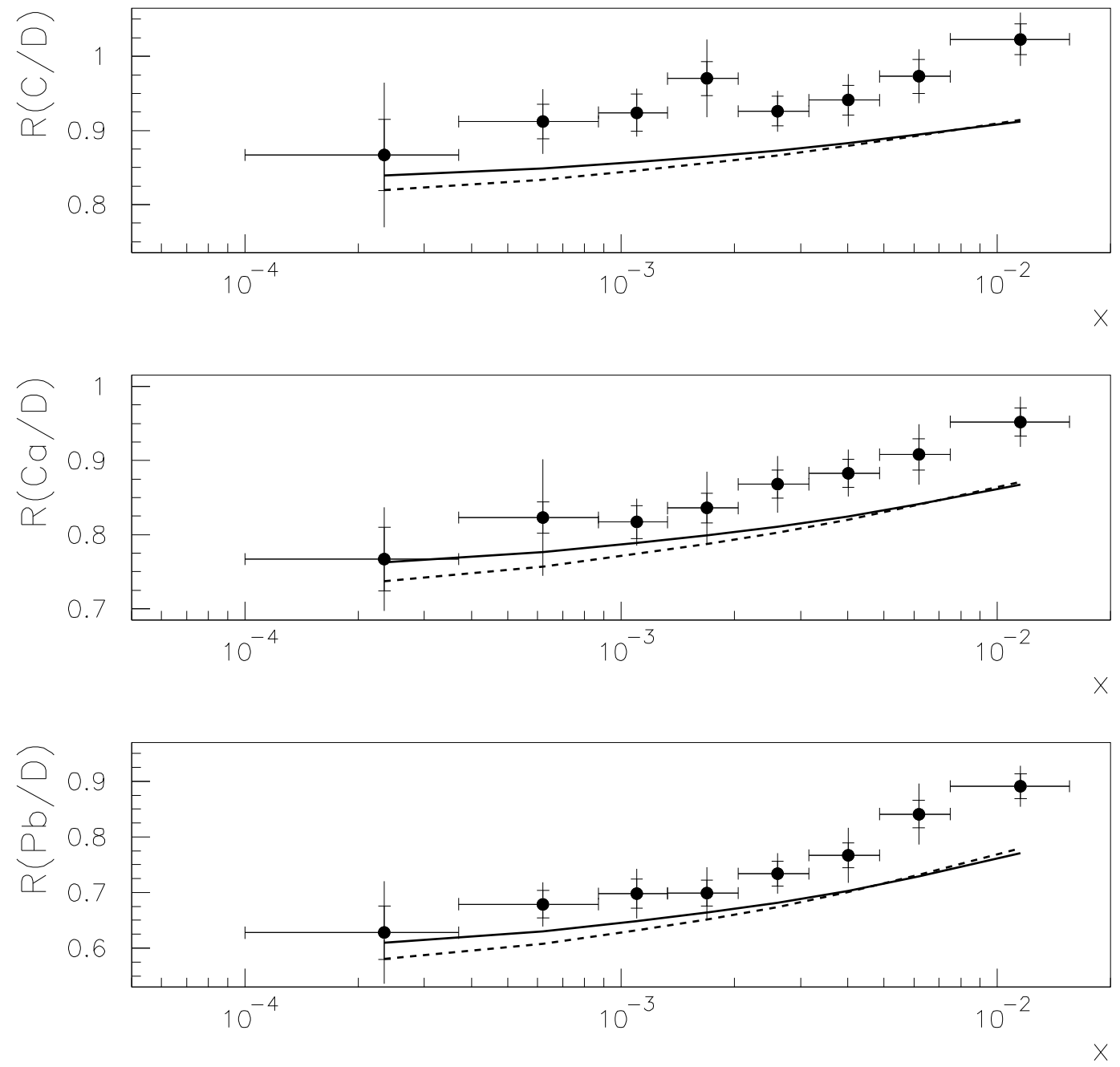

Fig. 1 

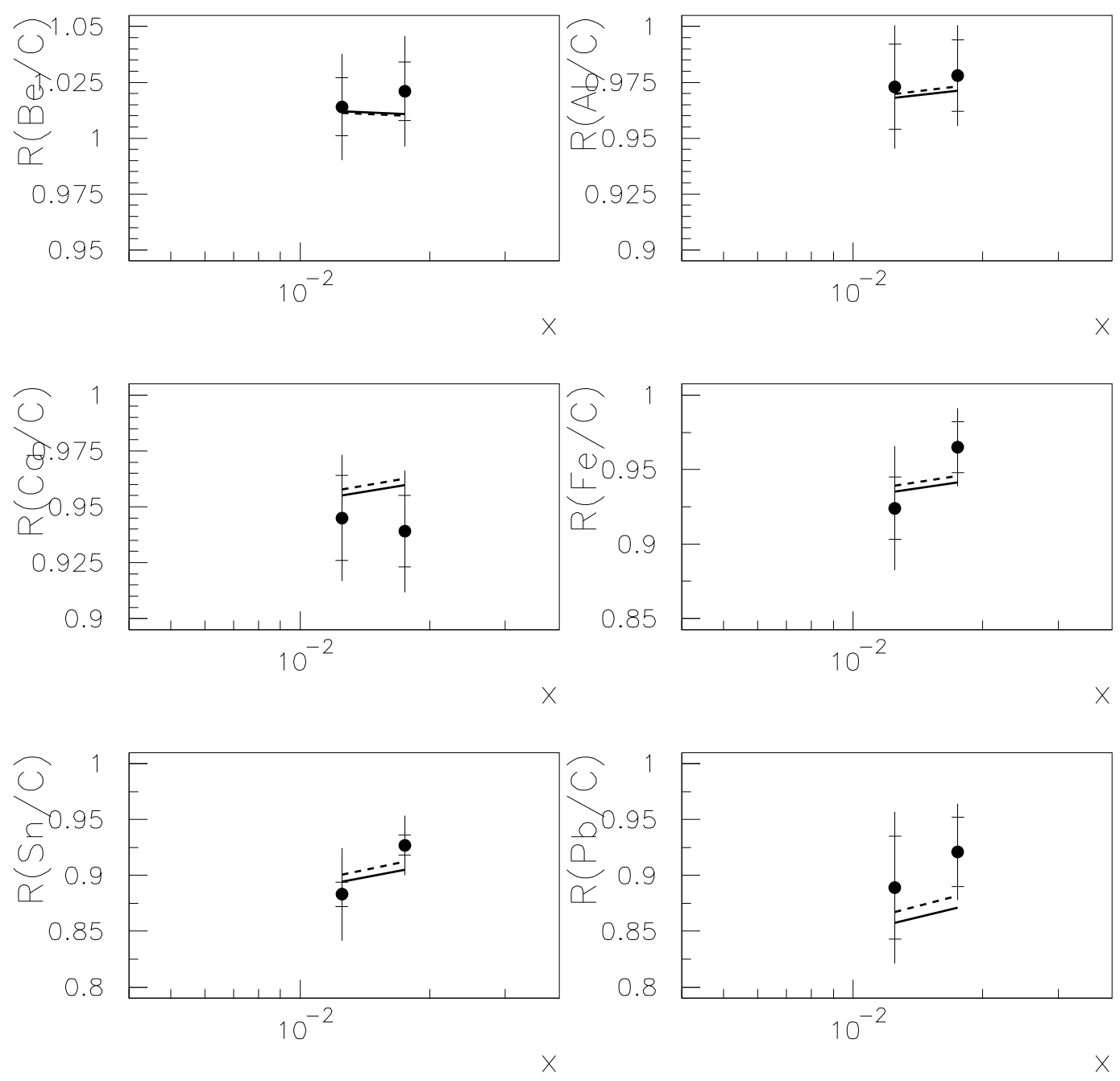

Fig. 2 

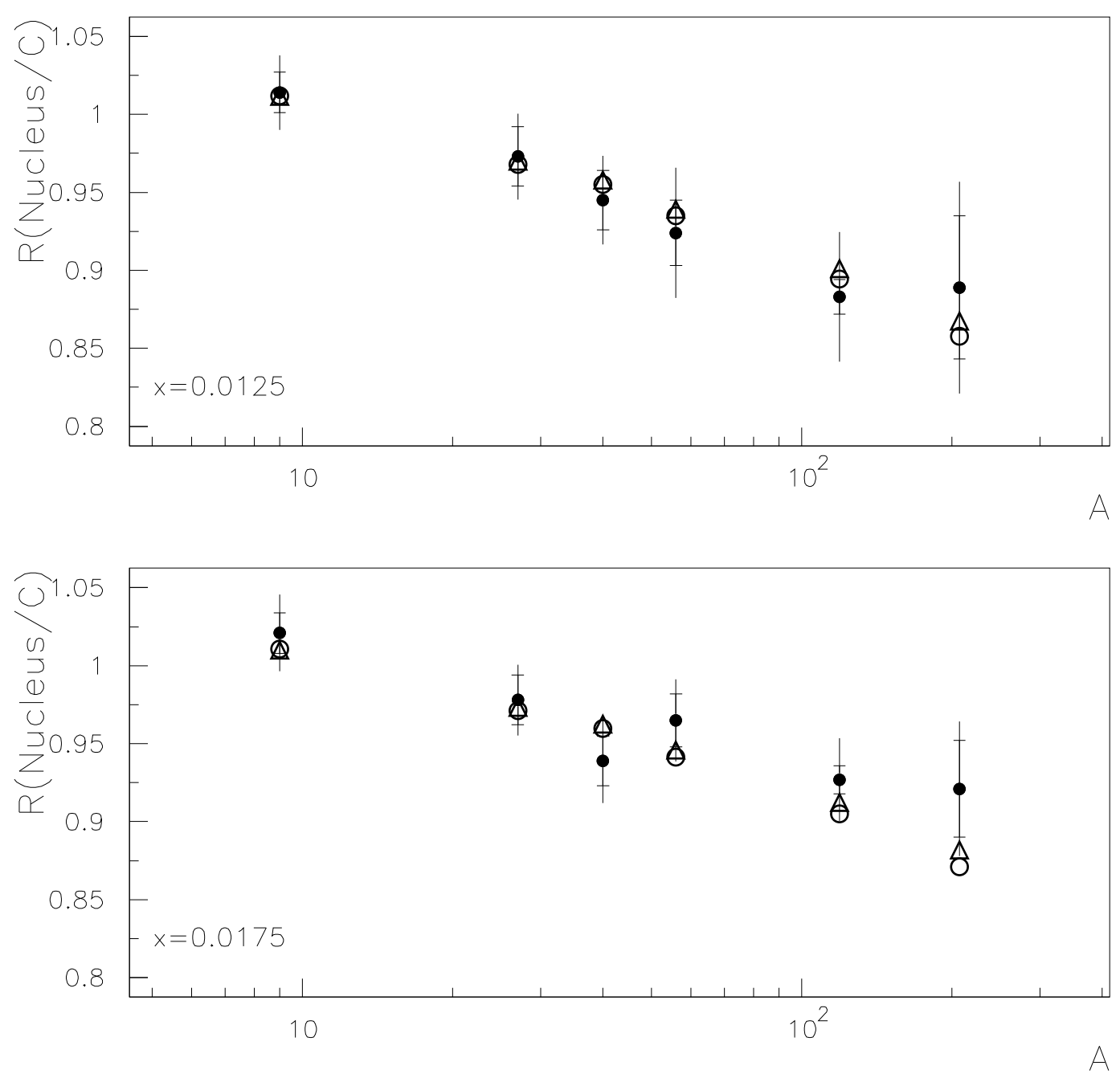

Fig. 3 

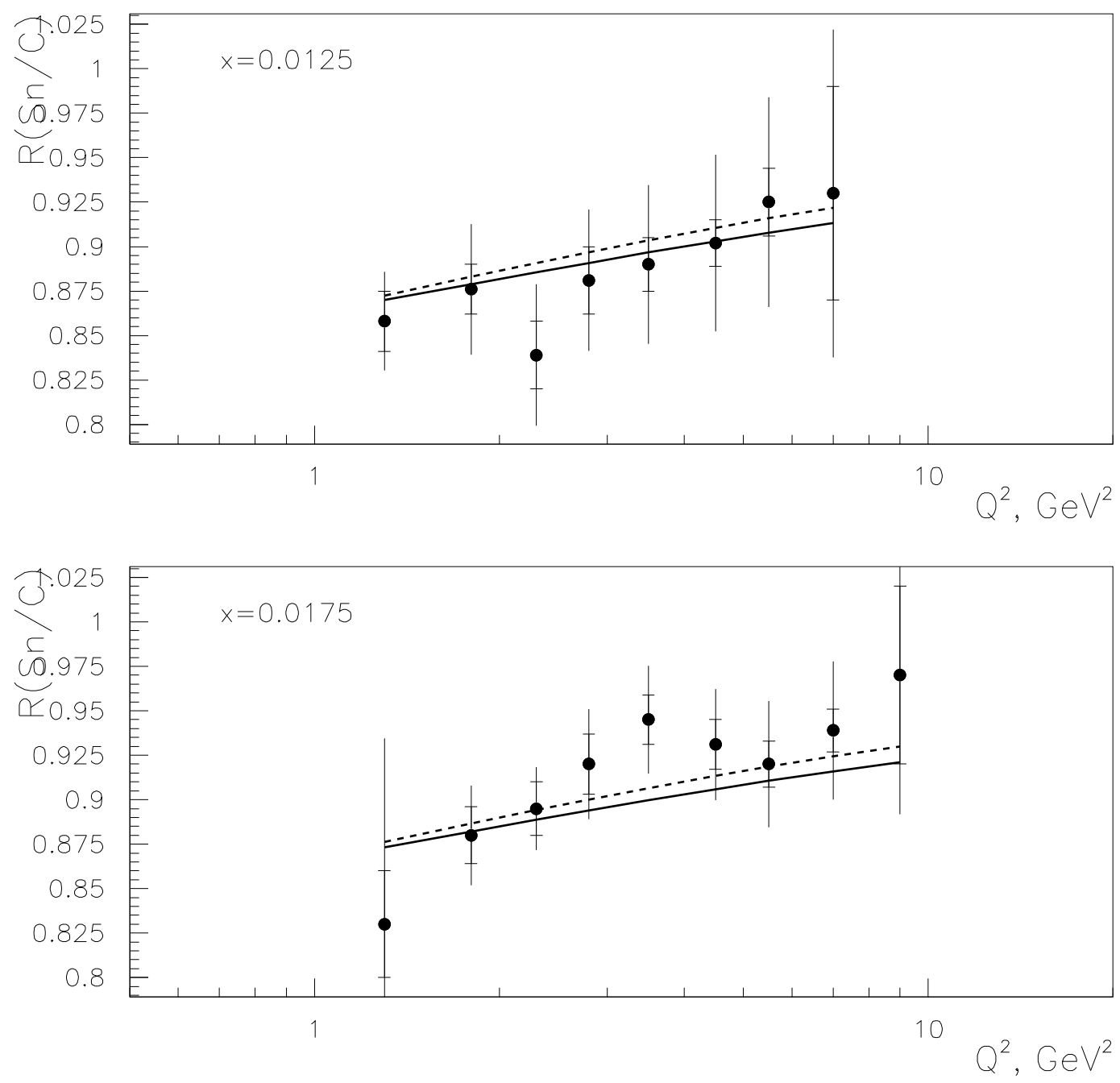

Fig. 4 

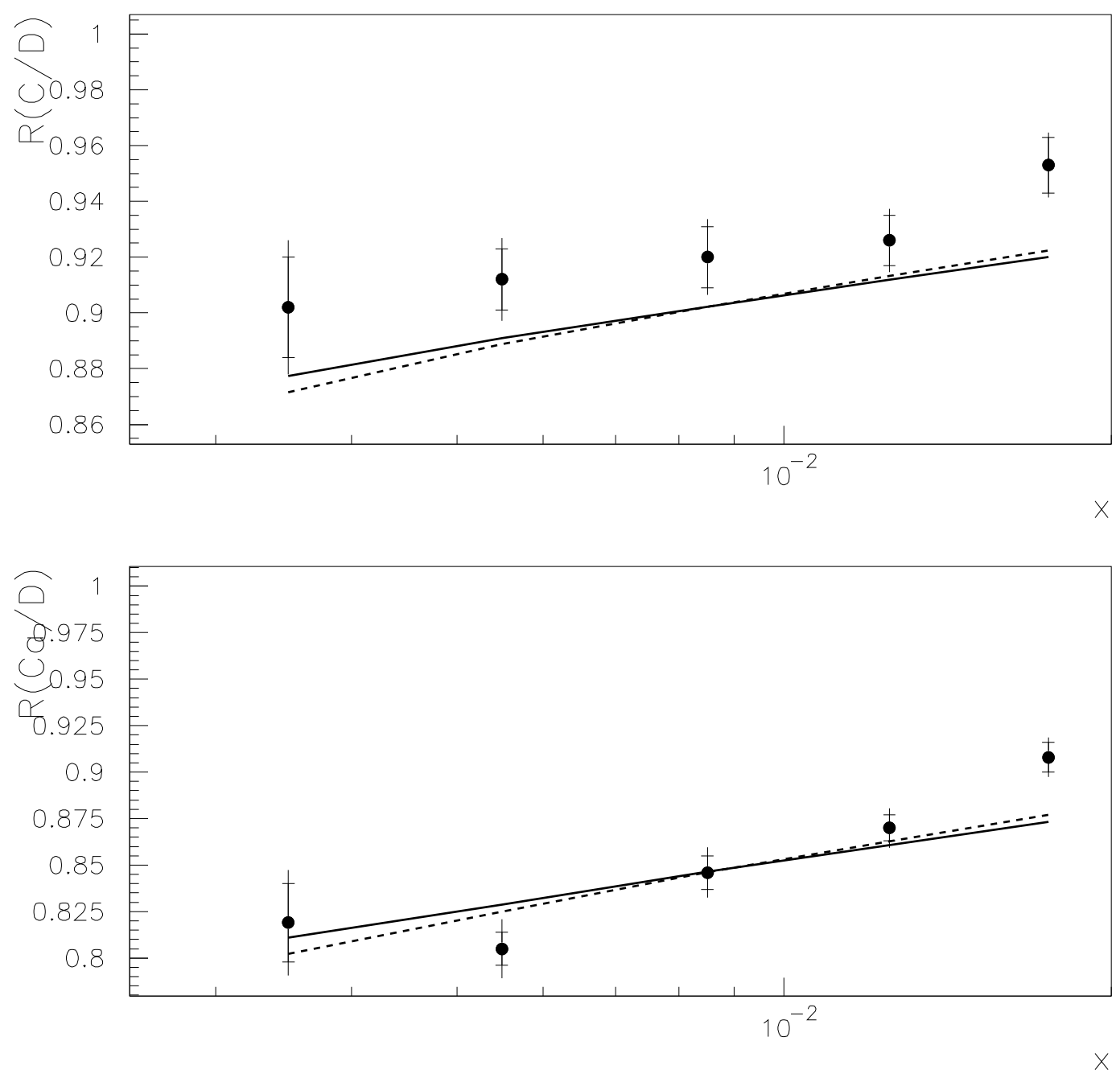

Fig. 5 

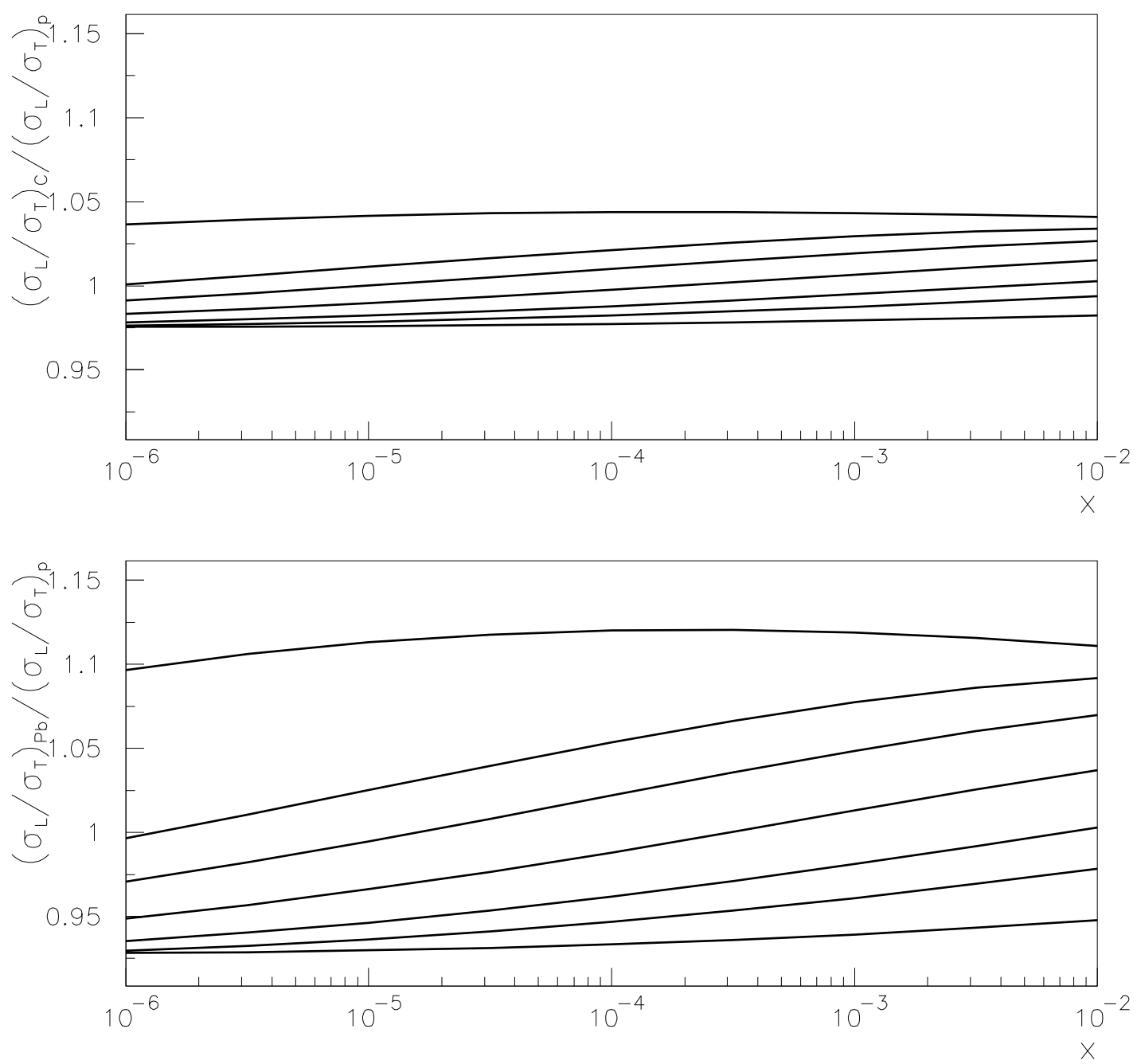

Fig. 6 

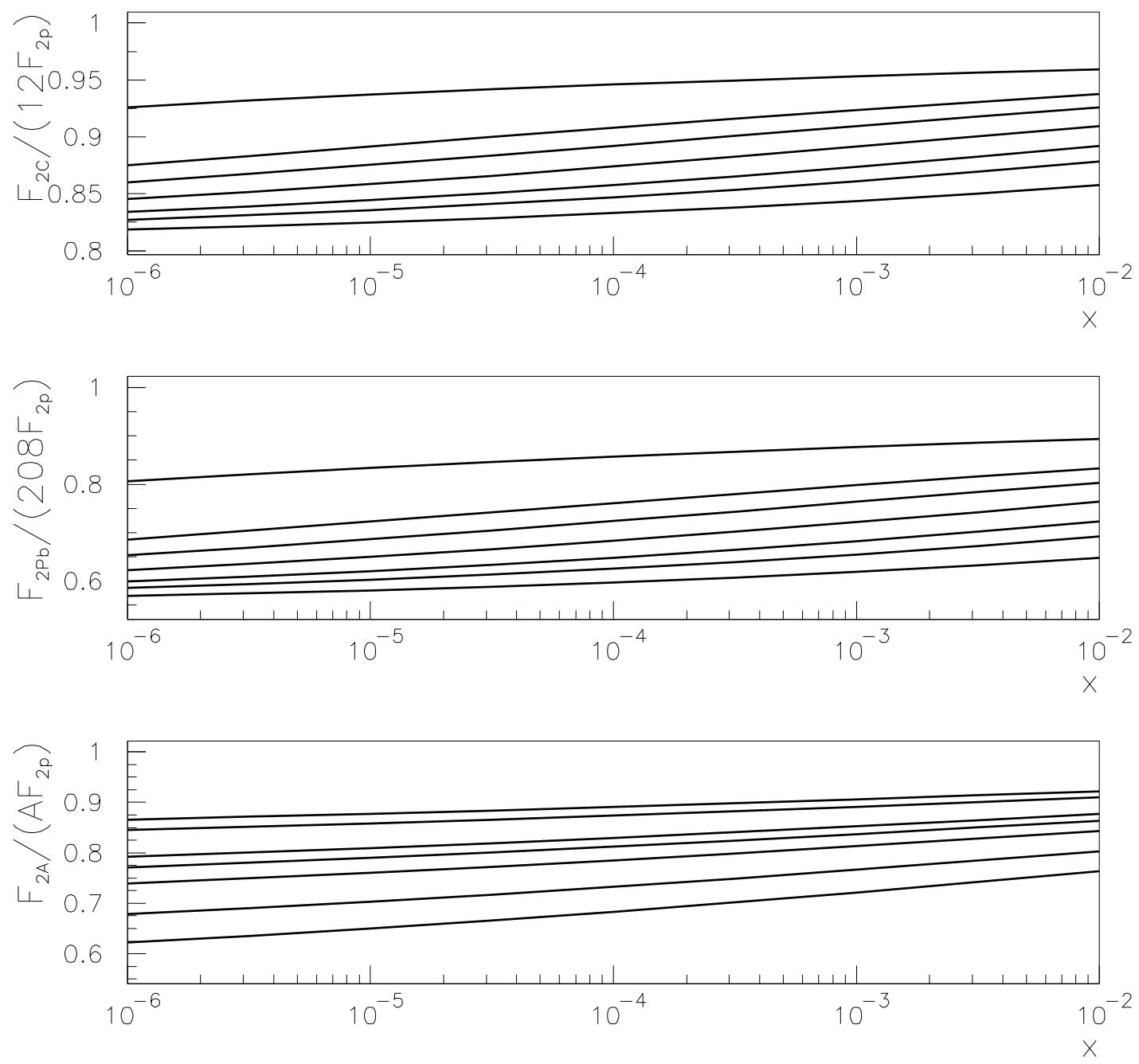

Fig. 7 

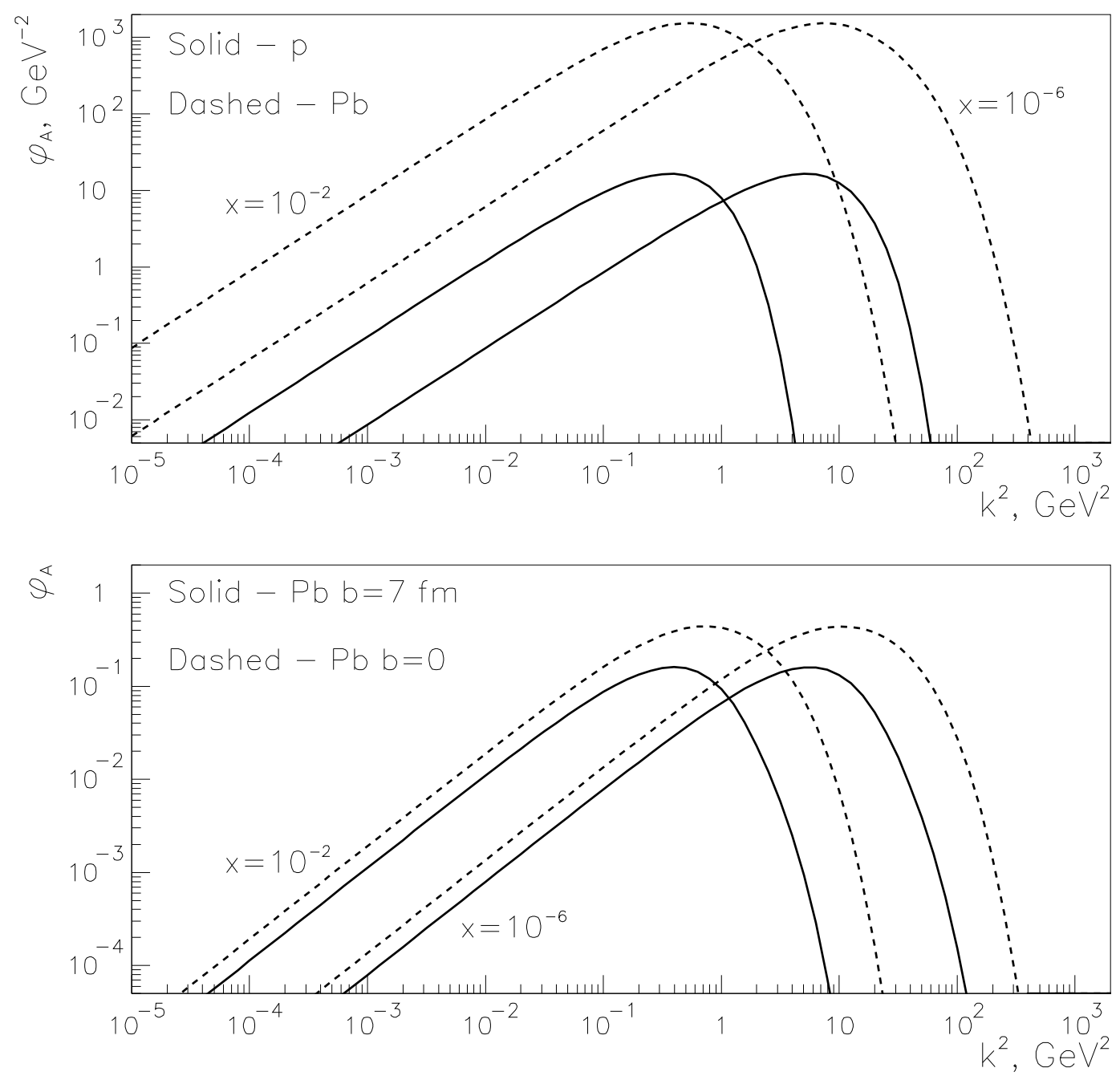

Fig. 8 

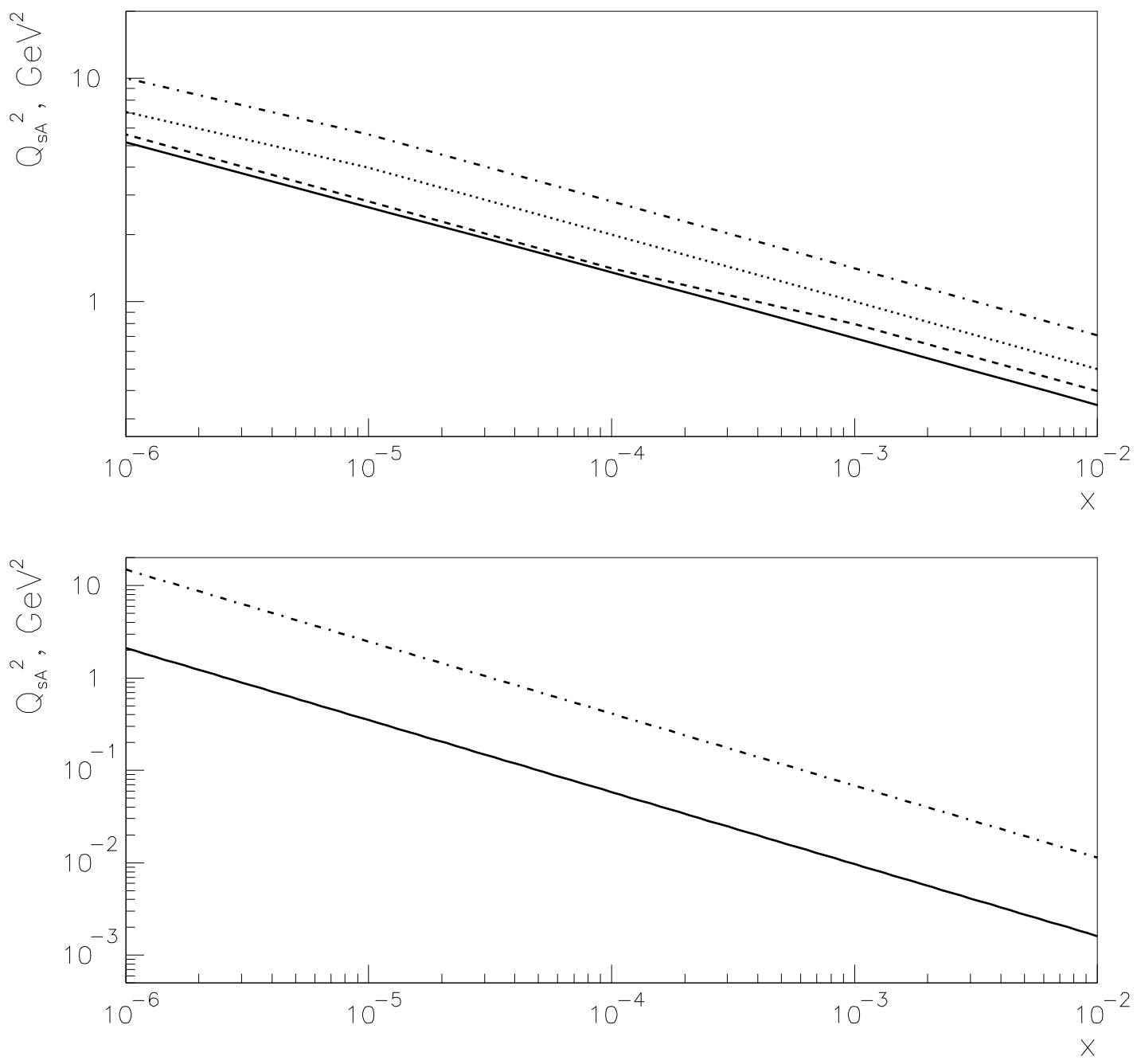

Fig. 9 